LUNG CANCER

\title{
Screening for lung cancer using low dose CT scanning: results of 2 year follow up
}

\author{
R MacRedmond, G McVey, M Lee, R W Costello, D Kenny, C Foley, P M Logan, on behalf of the \\ PALCAD investigators
}

Thorax 2006;61:54-56. doi: 10.1136/thx.2004.037580

See end of article for authors' affiliations

.....................

Correspondence to: Dr R E MacRedmond Department of Medicine, Education and Research Centre, Beaumont Hospital, Dublin 9, Ireland; rmacredmond@rcsi.ie

Received

16 November 2004

Accepted 15 April 2005

\begin{abstract}
Background: Screening with low dose chest computed tomographic scanning (LDCCT) may improve survival by identifying early asymptomatic lung cancer.

Methods: Four hundred and forty nine high risk subjects were screened with serial LDCCT scanning over 2 years. Fine needle aspiration biopsy was recommended for non-calcified nodules (NCNs) of $>10 \mathrm{~mm}$ diameter or demonstrating interval growth.

Results: NCNs were identified in 111 subjects (24.7\%), three of which were lung cancer. The overall prevalence $(0.4 \%)$ and incidence $(1.3 \%)$ rates of lung cancer detection were low. Three of the six lung cancers detected in the study were stage 1 non-small cell lung cancer; the remainder were unresectable central tumours. By contrast, eight subjects developed extrathoracic malignancy during the study period and other incidental pathology was noted in 221 subjects (49.2\%). Smoking cessation rates at 19\% were higher than in the general population, but $60.8 \%$ of subjects continued to smoke.

Conclusion: LDCCT scanning is useful in detecting early peripheral non-small cell lung cancers but its usefulness as a screening tool is limited by low specificity and by poor sensitivity for central tumours.
\end{abstract}

L ung cancer is the most common cancer worldwide and the leading cause of cancer related deaths in many Countries. ${ }^{1}$ The majority of patients present with advanced inoperable disease, but cure rates following surgery for early stage disease are excellent with 5 year survival rates of over $70 \%$ following resection of stage I non-small cell lung cancer (NSCLC). ${ }^{2}$ A screening method that reliably identifies early stage cancers could potentially improve mortality.

Much recent work has focused on the value of low dose chest CT (LDCCT) scanning as a screening tool for detecting early asymptomatic lung cancers. In particular, the work of the ELCAP group showed that LDCCT scanning is more sensitive than chest radiography in detecting small pulmonary nodules. They reported a prevalence rate of lung cancer of $2.7 \%$ in a high risk population and an incidence of lung cancer of $0.9 \%$ on repeat screening using LDCCT. ${ }^{3}$ In contrast, we recently reported data from baseline screening of 449 high risk individuals which identified only one operable lung cancer $(0.2 \%)$, higher rates of benign intervention, and high rates of ancillary pathology. ${ }^{4}$ Here we present follow up data from 2 years repeat screening of this cohort.

\section{METHODS}

Four hundred and forty nine subjects of median age 56.4 years (range 50-74) with a minimum smoking history of 10 pack years (mean 53.4) and still smoking at age 45, no prior history of cancer, and medically fit for thoracic surgery were recruited. Spiral $10 \mathrm{~mm}$, pitch 2, low dose (50 MA or less) CT images were obtained and reconstructed using a high resolution algorithm in overlapping $5 \mathrm{~mm}$ increments. The results of the prevalence scan determined intervention or repeat screening as previously described. ${ }^{4}$ Briefly, noncalcified nodules (NCNs) $<10 \mathrm{~mm}$ in diameter were followed with LDCCT scans at 6, 12 and 24 months, larger nodules or those showing interval growth were referred for fine needle aspiration biopsy (FNAB), and annual repeat LDCCT scanning was recommended where no nodule was seen on prevalence scanning.

\section{RESULTS}

A total of 1371 scans were performed on 449 subjects in the 2 year period. Twenty two subjects $(4.9 \%)$ withdrew from the study during the 2 years and a further $14(3.1 \%)$ did not complete because of intercurrent illness or death; 413 subjects $(92 \%)$ completed the 2 year repeat screening. The results are presented in table 1 .

Non-calcified nodules were identified in 93 subjects on the prevalence scan. A further 12 subjects were found to have nodules on repeat scanning which, in retrospect, were visible on the prevalence scan. Nodules measuring less than $10 \mathrm{~mm}$ on prevalence scanning were followed without change for 24 months in 97 of these 105 subjects; four did not complete the study, two were resected at the initial presentation (one NSCLC stage 1 and one hamartoma), and two demonstrated nodule growth.

Both cases of nodule growth were observed on the second interval scan. FNAB revealed no malignant cells in either subject. A spiculated appearance on the CT scan was suspicious for malignancy in one nodule with interval growth from $8 \mathrm{~mm}$ to $12 \mathrm{~mm}$, and lobectomy was performed revealing adenocarcinoma stage 1 . The other case with interval growth from $4 \mathrm{~mm}$ to $10 \mathrm{~mm}$ had a negative positron emission tomographic (PET) scan and no further interval growth on the follow up LDCCT scan so was deemed benign.

Six subjects were found to have new nodules during the 2 year follow up period, of which one proceeded to lobectomy revealing a neuroendocrine tumour. The other five subjects continue to be followed according to the study protocol and none has shown interval growth to date.

Three subjects had lung cancer detected in the mediastinum or hilum. One was identified on the prevalence scan and another had a small cell carcinoma and liver metastases at annual incidence screening. The third presented with

Abbreviations: FNAB, fine needle aspiration biopsy; LDCCT, low dose chest computed tomography; NCN, non-calcified nodule; NSCLC, nonsmall cell lung cancer 
Table 1 Rates of non-calcified nodules and lung cancer detected during the 2 year study period

\begin{tabular}{lrl}
\hline & N & $\%$ \\
\hline NCN on prevalence scan & 105 & 20.7 \\
NCN stable at 2 years & 97 & 21.6 \\
NCN growth on incidence scan & 2 & 0.4 \\
NSCLC stage 1 & 1 & 0.2 \\
New NCN on incidence scan & 6 & 1.3 \\
Neuroendocrine tumour & 1 & 0.2 \\
Lung cancer detected in 2 years & 6 & 1.3 \\
Prevalence lung cancer & 2 & 0.4 \\
NSCLC stage 1 & 1 & 0.2 \\
Small cell lung cancer & 1 & 0.2 \\
Incidence lung cancer & 3 & 0.7 \\
NSCLC stage 1 & 1 & 0.2 \\
Neuroendocrine tumour & 1 & 0.2 \\
Small cell lung cancer & 1 & 0.2 \\
Interval lung cancer & 1 & 0.2 \\
\hline NCN, non-calcified nodule; NSCLC, non-small cell lung cancer. \\
The values are the numbers of individual abnormalities and the \\
percentage represents the proportion of the total study population with \\
this finding.
\end{tabular}

paraneoplastic cerebellar syndrome 5 months after a normal prevalence scan and had a large central lung mass with lymphadenopathy. Bronchoscopy, mediastinoscopy and FNAB were non-diagnostic. The patient suffered a myocardial infarction precluding further intervention and was palliated.

By comparison, over the 2 years of the study eight subjects (1.6\%) were diagnosed with an extrathoracic malignancy (two pancreatic, two brain, one breast, one ovarian, one renal, and one laryngeal). Unlike the Mayo Clinic group who reported incidental detection of extrathoracic malignancy in $7.9 \%$ of their subjects as a favourable byproduct of screening, ${ }^{5}$ none of these cancers was identified by LDCCT scanning in our cohort. Other significant pathology was noted in 221 subjects (49.2\%)-largely emphysema, bronchiectasis, and coronary artery disease-as previously reported. ${ }^{4}$

Three hundred and seven subjects (68.4\%) were current smokers at the start of the study. Smoking cessation advice was given by the study coordinator and reinforced at each follow up. Referral to a smoking cessation group was offered to all participants of whom only four (1.3\%) accepted. Fifty nine subjects (19.2\%) reported stopping smoking during the study period while five (1.6\%) restarted. We did not validate smoking status with expired carbon monoxide levels as selfreported abstinence has previously been shown to be very reliable. ${ }^{6}$ Our smoking cessation rate is comparable to the $14 \%^{6}$ and $23 \%^{7}$ quit rates reported by the Mayo group and a subset of the ELCAP cohort, respectively, and is higher than the $5-7 \%$ per year rates expected in the population. ${ }^{8}$ Greater motivation to quit smoking might be expected in a selfselected volunteer population, but these higher quit rates also probably reflect the effectiveness of such simple measures as smoking cessation advice and positive reinforcement. Despite demonstrating their concern regarding the harmful effects of smoking by participating in a screening programme, it is disappointing that 251 of 413 subjects $(60.8 \%)$ who completed the study did so while continuing to smoke.

None of the nodules progressed to advanced stage cancer within the screening interval, suggesting that our re-screening interval is appropriate. Five subjects $(1.1 \%)$ had invasive interventions for benign lesions triggered by baseline screening-one mediastinoscopy and four FNABs, one of which proceeded to lobectomy. A further FNAB for a benign lesion was triggered by nodule growth on interval screening. This was complicated by a pneumothorax. Selective use of PET in combination with CT scanning has been suggested as a means of reducing benign intervention but led to surgical biopsy for benign disease in three of five resections and false negative results in two cases of adenocarcinoma in the cohort of 1035 patients reported by Pastorino et al. ${ }^{9}$ FNAB, in contrast, resulted in no false positives and one false negative of five negative biopsies. Of four surgical resections in our cohort, only one was found to be a benign lesion.

\section{DISCUSSION}

Two years of screening with LDCCT scanning in a high risk population identified three resectable lung tumours $(0.7 \%)$ : one prevalence and two incidence cancers including one neuroendocrine tumour. A similar rate of inoperable cancer was observed with two small cell cancers and one interval unclassified cancer. In keeping with another similar study from Northern Europe, ${ }^{10}$ we failed to replicate the high rates of LDCCT detected early stage NSCLC reported by Henschke and the Cornell group. This may be partly attributable to the older age profile of the ELCAP group, which was on average a decade older than the other two groups, but also highlights the difficulty in applying results of screening in one population to another.

The proportion of small cell cancers $(33 \%)$ was higher than in our general population where small cell cancers comprise $17 \%$ of all cancers diagnosed in our institution. ${ }^{11}$ This overrepresentation is probably an artefact of our small sample size. Small cell lung cancer is an aggressive tumour characterised by rapid doubling time and early metastasis and, as such, should not be considered as a realistic target for LDCCT screening. Adenocarcinomas, in contrast, are typically peripheral and more indolent tumours, making them more amenable to detection by screening. Adenocarcinomas generally account for $30-40 \%$ of all lung cancers but were overrepresented in the total number of cancers detected in the cohorts studied by both Henschke et al ${ }^{12}(67 \%)$ and the Mayo Clinic $(60 \%) .{ }^{5}$ It remains to be proved that earlier diagnosis of slow growing adenocarcinomas will confer a real mortality benefit as survival benefit with indolent tumours is particularly subject to the effects of overdiagnosis and length-time bias. $^{13}$

Using an algorithm such as ours with selected use of FNAB, LDCCT scanning is useful for detecting early stage peripheral NSCLC. Its usefulness as a screening tool for lung cancer, however, is limited by poor sensitivity in detecting central endobronchial tumours and by the biologically aggressive nature of small cell lung cancer. In addition, the low specificity of LDCCT scanning necessitates follow up of a large number of nodules that are ultimately benign. The cost of population based LDCCT screening for lung cancer has been estimated at over \$100 000 per year of life gained, ${ }^{14}$ and reduction in mortality is yet to be proven. Our lower detection rates and high rates of ancillary pathology requiring follow up or intervention may indicate even higher costs. These may prove prohibitive, particularly when over half of the screening population continue to smoke.

\section{Authors' affiliations}

R MacRedmond, G McVey, R W Costello, Department of Medicine, Royal College of Surgeons in Ireland, Dublin, Ireland

M Lee, P M Logan, Department of Radiology, Royal College of Surgeons in Ireland, Dublin, Ireland

D Kenny, C Foley, Department of Clinical Pharmacology, Royal College of Surgeons in Ireland, Dublin, Ireland

This study was conducted in the Clinical Research Centre, Beaumont Hospital and funded in part by the Higher Education Authority, Ireland.

The PALCAD investigators comprise the authors and Dr E Kay, Professor $M$ Leader, Dr P Broe, Professor C Kelly, Dr L Grogan, Professor S O'Neill and Professor NG McElvaney. 


\section{REFERENCES}

1 Shibuya K, Mathers CD, Boschi-Pinto C, et al. Global and regional estimates of cancer mortality and incidence by site: II. Results for the global burden of disease 2000. BMC Cancer 2002;2:37

2 Flehinger BJ, Kimmel M, Melamed MR. The effect of surgical treatment on survival from early lung cancer. Implications for screening. Chest 1992:101:1013-8.

3 Henschke Cl, Yankelevitz DF, Libby DM, et al. Early Lung Cancer Action Project: annual screening using single-slice helical CT. Ann NY Acad Sci 2001;952:124-34.

4 MacRedmond R, Logan PM, Lee $M$, et al. Screening for lung cancer using low dose CT scanning. Thorax 2004;59:237-41.

5 Swensen SJ, Jett JR, Sloan JA, et al. Screening for lung cancer with low-dose spiral computed tomography. Am J Respir Crit Care Med 2002;165:508-13.

6 Cox LS, Clark MM, Jett JR, et al. Change in smoking status after spiral chest computed tomography scan screening. Cancer 2003;98:2495-501.

7 Ostroff JS, Buckshee N, Mancuso CA, et al. Smoking cessation following CT screening for early detection of lung cancer. Prev Med 2001;33:613-21.
8 Smith SS, Fiore MC. The epidemiology of tobacco use, dependence, and cessation in the United States. Primary Care 1999;26:433-61.

9 Pastorino U, Bellomi M, Landoni C, et al. Early lung-cancer detection with spiral CT and positron emission tomography in heavy smokers: 2-year results. Lancet 2003;362:593-7.

10 Diederich S, Thomas M, Semik M, et al. Screening for early lung cancer with low-dose spiral computed tomography: results of annual follow-up examinations in asymptomatic smokers. Eur Radiol 2004;14:691-702.

11 Logan PM. Lung cancer audit in an Irish teaching hospital. Ir J Med Sci 2004; 173:7-8.

12 Henschke Cl, McCauley DI, Yankelevitz DF, et al. Early Lung Cancer Action Project: overall design and findings from baseline screening. Lancet 1999:354:99-105

13 Patz EF Jr, Goodman PC, Bepler G. Screening for lung cancer. N Engl J Med 2000;343:1627-33

14 Mahadevia PJ, Fleisher LA, Frick KD, et al. Lung cancer screening with helical computed tomography in older adult smokers: a decision and costeffectiveness analysis. JAMA 2003;289:313-22.

\section{LUNG ALERT}

\section{The public health burden of acute lung injury}

$\Delta$ Rubenfeld GD, Caldwell E, Peabody E, et al. Incidence and outcomes of acute lung injury. N Engl J Med 2005;353:168593

7

he public health burden of acute lung injury (ALI/ARDS) in terms of morbidity, mortality, length of hospitalisation, and need for rehabilitation is poorly appreciated.

This study reports the results of a prospective, population based, cohort study conducted in 21 hospitals in and around King County, Washington, USA to determine the incidence and mortality associated with ALI/ARDS. Between April 1999 and July 2000, 4251 adults requiring mechanical ventilation for more than 24 hours were screened. Of the 1687 patients with acute hypoxaemic respiratory failure, 1113 had ALI, 828 of whom (74\%) had ARDS. The crude incidence of ALI/ARDS was 78.9/58.7 cases per 100000 person-years, respectively, giving age adjusted national estimates of 86.2/64 cases per 100000 . The inhospital mortality was $38.5 \%$ for ALI and $41.1 \%$ for ARDS. Mortality increased with age, reaching $60 \%$ for patients aged 85 or older. The calculated incidence of ALI/ARDS in this study was 2-40 times higher than previously reported with an estimated 190600 new cases of ALI every year requiring 3.6 million hospital days, 2.15 million ICU days, and leading to 74500 deaths. This number is comparable to the deaths due to breast cancer or HIV in the USA in 1999. Of note, only $34 \%$ of the survivors were discharged directly home while $51 \%$ were transferred to rehabilitation or nursing facilities.

This study provides us with a better understanding of the epidemiology of ALI/ARDS which is useful for allocating future ICU resources and planning physical and neurocognitive rehabilitation programmes for ICU survivors.

L Camporota Research Registrar, Guy's \& St Thomas' NHS Foundation Trust, London, UK; luigicamporota@yahoo.co.uk 